Meta

Journal des tradlucteurs

Translators' Journal

\title{
Traduction et informatique : perspectives pour les années $\mathbf{8 0}$
}

\section{Wallace Schwab}

Volume 26, numéro 1, mars 1981

L'informatique au service de la traduction

Machine Aids to Translation

URI : https://id.erudit.org/iderudit/004062ar

DOI : https://doi.org/10.7202/004062ar

Aller au sommaire du numéro

Éditeur(s)

Les Presses de l'Université de Montréal

ISSN

0026-0452 (imprimé)

1492-1421 (numérique)

Découvrir la revue

Citer cet article

Schwab, W. (1981). Traduction et informatique : perspectives pour les années 80. Meta, 26(1), 48-56. https://doi.org/10.7202/004062ar d'utilisation que vous pouvez consulter en ligne.

https://apropos.erudit.org/fr/usagers/politique-dutilisation/ 


\title{
Traduction et informatique : perspectives pour les années 80
}

\author{
WALLACE SCHWAB
}

La présence croissante de l'informatique dans toutes les sphères d'activités humaines crée un besoin de réévaluer à la lumière de cette nouvelle technique les démarches cognitives et les moyens physiques utilisés dans de nombreuses professions libérales. Dans la présente étude ${ }^{1}$, nous tentons de démontrer les mutations que la traduction sera appelée à subir comme conséquence de la révolution télématique.

Il s'agit donc de voir quels outils la médiatique peut offrir à la traduction, comment l'informatique s'implante partout et de quelle manière le milieu du travail en traduction n'échappera pas aux bouleversements provoqués par ces nouvelles techniques.

Notre préoccupation en la matière rejoint, entre autres, la pensée des chercheurs de l'Université Carnegie-Mellon :

Il serait souhaitable que des efforts fussent entrepris pour élaborer un système de traduction assistée par ordinateur qui permettrait à un homme de dialoguer, en accès direct (...), avec un dictionnaire et d'autres aides à la recherche telles que la visualisation et la mise en forme, pour produire des traductions de haute qualité avec une réduction importante de temps et d'efforts par rapport aux méthodes traditionnelles des traductions humaines $^{2}$.

Les contributions de l'informatique à la traduction sont déjà impressionnantes et pour recenser dans leur ensemble les avantages qu'elle procure à la traduction, il convient de les regrouper en deux catégories générales : les réalisations existantes dont on tire profit actuellement et celles qui verront le jour au cours de la prochaine décennie.

La terminologie automatisée constitue, sans contredit, une des percées les plus marquantes des aides informatisées à la traduction. À l'Université du Québec, les traducteurs bénéficient déjà depuis plus d'un an des ressources multiples de la banque de terminologie PLATERM du système PLATON ${ }^{3}$. Au

1. Le présent article résume certaines idées exprimées par l'auteur dans un document intitulé Projet visant la création d'un laboratoire de traduction et d'adaptation de logiciel, coll. : Communications, Rapports de Recherche, ${ }^{\circ} 21$, Sainte-Foy, Université du Québec, 1980.

2. Carestia-Greenfield, Concetta et Daniel Serain, la Traduction assistée par ordinateur; des banques de terminologie aux systèmes interactifs de traduction, Paris, AFTERM, août 1979, p. 80 .

3. Schwab, Wallace et Richard St-Denis, «PLATERM : la banque de terminologie du système PLATON $»$, in META, Journal des traducteurs, vol. 25, $\mathrm{n}^{\circ}$ 3, septembre 1980. 
Gouvernement du Québec, l'Office de la langue française dessert sa vaste clientèle à l'aide d'une banque de terminologie informatisée qui contient plus de 400000 fiches de terminologie interrogeables ${ }^{4}$. Le Secrétariat d'État du Gouvernement fédéral compte aujourd'hui plus d'un million d'entrées dans son répertoire terminologique ${ }^{5}$.

Les banques de terminologie sont susceptibles d'offrir des possibilités que les dictionnaires ne peuvent pas donner. Ainsi, l'utilisation de programmes permet non seulement de stocker, trier et classer des termes nouveaux, mais encore de parcourir un article de journal, d'y sélectionner des termes techniques et d'en produire une liste suivant l'ordre d'apparition, en donnant pour chacun l'équivalent dans la langue d'arrivée. Erhard Lippmann appelle ces listes des «condensés terminologiques»...

$\mathrm{Au}$ cours d'une expérience, les traducteurs utilisant ce genre de listes ont gagné le tiers du temps habituellement nécessaire et n'ont fait que deux tiers des erreurs commises par les traducteurs se servant des aides habituelles à la traduction, comme les dictionnaires techniques imprimés...

La banque de terminologie permet également la composition immédiate de glossaires spécialisés de termes relatifs à un domaine ou sous-domaine. Cette possibilité est intéressante pour ceux qui désirent obtenir un glossaire dans des domaines spécialisés. Une banque de terminologie est semblable à un dictionnaire dont les pages peuvent diminuer ou augmenter, se contracter ou s'étendre en fonction des besoins immédiats des utilisateurs ${ }^{6}$.

Parallèlement aux activités des traducteurs et des linguistes, les juristes québécois et canadiens ont également contribué largement à la recherche en terminologie informatisée, sous forme de diverses bases de données. À l'Université d'Ottawa, il existe une unité de recherche appelée $\mathrm{JURIVOC}^{7}$ qui est rattachée à la Faculté de droit. On y fait le recensement, par repérage informatisé, de tous les syntagmes bilingues reconnus pour leur valeur juridique dans les lois fédérales. Un échantillon de la capacité de cette banque qui se compose de 1001 termes a fait l'objet d'un premier lexique bilingue ${ }^{8}$. Depuis 1970 , à la Société québécoise d'information juridique (SOQUIJ), on étudie la construction de thésauri bilingues en vue de faciliter le repérage des arrêts de la jurisprudence québécoise en langues anglaise et française ${ }^{9}$. À l'extérieur du Québec, on peut souligner rapidement les travaux entrepris par le système KWIC Law en Ontario, par le CEDIJ en France et, dans les Communautés européennes, par la banque de terminologie polyglotte du Luxembourg où l'on a mis sur pied une base de données fort ambitieuse pour satisfaire des demandes très variées.

Nous pouvons donc en conclure que la banque de terminologie informatisée constitue pour l'avenir un instrument de travail indispensable que les pra-

4. Chiffre fourni par Mme Tina Célestin de la Banque de terminologie du Québec.

5. Selon les données fournies par la Banque de terminologie du Secrétariat d'État.

6. Op. cit., Carestia-Greenfield, Concetta et al., p. 3.

7. Bergeron, Viateur et al., Lexicographie, bilinguisme juridique et ordinateur, Ottawa, Les Éditions de l'Université d'Ottawa, 1976, $330 \mathrm{p}$.

8. Bergeron, Viateur et al., Un lexique bilingue de 1001 mots extraits de 24 chapitres des statuts révisés du Canada, Ottawa, Université d'Ottawa, 1975, 324 p.

9. Schwab, Wallace J., "La réalisation du thésaurus-s et du thésaurus-g ", in la Revue juridique Thémis (1971), 6 R.J.T., n ${ }^{\circ} 1$, p. 69-84.; Note: avant 1976, SOQUIJ était connue sous le nom de DATUM/SEDOJ. 
ticiens de la traduction utiliseront au même titre que leurs dictionnaires traditionnels.

À l'instar des banques de terminologie, la documentation automatisée représente une autre innovation technique au service de la traduction. Au Québec, il existe un exemple d'un tel système à l'Office de la langue française, où le répertoire informatisé de documents «Terminoq II» renseigne ses interrogateurs sur le contenu terminologique des ouvrages dépouillés par les terminologues de cet organisme ${ }^{10}$.

Une partie non négligeable de la documentation automatisée doit être consacrée à l'archivage et au repérage des documents traduits. Cela s'explique par le fait que lorsque le traducteur est appelé à faire de nombreuses traductions dans un même domaine, il lui arrive de revoir les mêmes problèmes terminologiques, syntaxiques... et parfois les mêmes textes! Ce phénomène se produit souvent dans les domaines techniques, où les mises à jour de manuels, par exemple, peuvent entraîner la retraduction d'un texte à de nombreuses reprises. Que de dédoublements d'efforts éliminés lorsqu'on peut revoir la dernière traduction faite, qu'elle remonte à une semaine, un mois ou un an!

Parmi les nouveautés qu'on trouve dans les cabinets de traduction depuis les trois ou quatre dernières années, les machines de traitement de textes, qui se présentent comme un clavier de machine à écrire relié à un écran de télévision, constituent les premiers indices d'une "révolution tranquille» qui produira des effets à tous les stades du processus de la traduction. Sous sa forme la plus primitive, la machine de traitement de textes ne représente qu'une aide raffinée à la disposition des secrétaires. Toutefois, ses dispositifs électroniques permettront de plus en plus la préparation intégrale d'un document dans un milieu informatisé. Cela signifie, entre autres choses, une réduction de l'usage du papier, la correction instantanée des épreuves, la suppression des deuxième et troisième versions dactylographiées d'un même document ainsi qu'une accélération du travail de bureau. On apprécie particulièrement dans ces machines, les fonctions permettant la recherche et la substitution des chaines de caractères, et la transposition de sections de texte d'un endroit à un autre au sein d'un même document. Selon les nombreux traducteurs et chefs de service que nous avons consultés ${ }^{11}$, ces instruments font désormais partie de l'arsenal moderne du traducteur, encore au même titre que le dictaphone et la machine à écrire, mais il semble bien qu'ils soient appelés à remplacer cette dernière.

Pourquoi tant d'insistance sur l'aspect «révolutionnaire» des techniques de traitement de mots? Sans doute parce qu'elles conduisent insensiblement à long terme vers une automatisation de plus en plus complète du processus de la traduction. Aujourd'hui, dans le commerce, on fait une distinction entre les

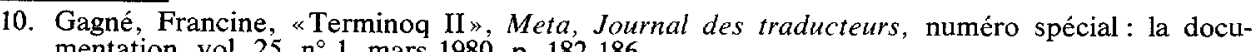
mentation, vol. $25, \mathrm{n}^{\circ} 1$, mars 1980 , p. 182-186.

11. L'auteur tient à remercier chaleureusement: M. Yvan Bouvier, directeur du service de traduction au Secrétariat d'État ainsi que son personnel; M. Michel Cardin, directeur à Montréal du bureau régional de traduction au Secrétariat d'État; $M$. Jean-Pierre Quijano, directeur de Technitrans Ltée; M. Marc Nakache, traducteur en chef à AES DATA Ltée de Montréal, du temps et des renseignements qu'ils ont si généreusement accordés. 
machines de traitement de textes et les appareils de traitement de données. Toutefois, cette distinction disparaîtra au cours des prochaines années au profit des systèmes intégrés d'information ${ }^{12}$ qui traiteront indifféremment textes et données selon la compétence de l'usager. Cet événement affectera le processus de la traduction du fait que celui-ci se divise en diverses étapes relevant tantôt du traitement de textes, tantôt du traitement de données. Actuellement, les ressources informatiques se trouvent éparpillées à travers nombre de systèmes qui ne sont pas nécessairement compatibles entre eux. C'est ainsi que lors de la traduction d'un texte, il se peut que le traducteur doive d'abord consulter une banque de terminologie souvent par l'intermédiaire d'un terminologue préposé au terminal, puis emmagasiner son texte dans un autre système informatique et, enfin, recourir à un troisième système pour faire imprimer le produit fini et le transmettre au client. Si, à son tour, le client préfère une version informatisée à la copie imprimée, il n'est pas certain, à l'heure actuelle, qu'un simple transfert de fichiers permette d'effectuer une livraison électronique de la marchandise. Bref, la démarche ci-dessus fait appel à trois systèmes informatiques au lieu d'un seul et unique canal électronique efficace, et même s'il s'agit de fichiers informatisés, il n'en demeure pas moins que c'est du gaspillage.

Voici donc quelques innovations qui sont devenues des pratiques courantes dans le domaine de la traduction et cette mutation ne vient que de commencer.

À première vue, le deuxième volet peut projeter une image de «futurologie débridée». Or, il n'en est rien. Certes, les extrapolations qui suivent représentent une évolution possible dont la réalisation peut être en cours ou n'être pas encore amorcée. Cette prévision tente de dégager un ensemble d'innovations plausibles et, dans certains cas, de réunir sous l'éclairage des besoins traductionnels, des techniques existantes bien que dispersées, ce qui peut conduire à la découverte d'applications nouvelles pour une technologie disponible et éprouvée. Il s'agit donc ici de mettre en relief l'optimalisation du rendement du processus de la traduction à l'aide d'instruments électroniques.

Arrêtons-nous d'abord à la fonction statistique du système informatique; rien n'est plus facile pour un ordinateur que de compter le nombre de mots contenus dans un texte, par exemple, à condition bien entendu que le texte doit cléjà entré en mémoire. Dans l'établissement de ses honoraires, le traducteur apprécie d'emblée le décompte informatisé des mots contenus dans le texte de départ. Que de travail fastidieux éliminé ! Mieux encore, pour supprimer la contrainte voulant que le texte soit déjà en mémoire, au service de traduction du Secrétariat d'État, on a demandé aux fournisseurs de matériel de mettre au point un «compte-mots». Aujourd'hui, cette demande est devenue une réalité et l'appareil en question fonctionne selon les principes du lecteur optique. Il recense de façon approximative le nombre de mots contenus dans une page de texte et livre les résultats de ses «cogitations» sur un bout de papier, à la

12. Wohl, Amy, «A Review of Office Automation», in DATAMATION, fév. 1980, vol. 26, $\mathrm{n}^{\circ} 2$, p. 116-119. 
manière des caisses enregistreuses dans les grands magasins. La version actuelle présente certaines lacunes, notamment le fait que son bon fonctionnement exige des pages imprimées qui soient impeccables, mais un modèle amélioré est prévu. L'évolution de cet appareil conduira tôt ou tard à la lecture optique du texte entier, opération ayant pour résultat l'entrée en mémoire du texte, le décompte de ses mots, le tri et la recherche terminologique. Dès lors, il devient évident que l'entrée du texte de départ en mémoire comporte des répercussions intéressantes pour les nombreuses étapes du processus de la traduction. On sait que le traducteur, saisi d'un nouveau texte, doit en premier lieu recenser tous les termes susceptibles de freiner son travail de traduction. Ce recensement se fait ordinairement par un repérage manuel lors de la première lecture du texte. Il s'agit d'un processus répétitif qui suit tous les méandres du texte de façon linéaire et qui n'offre aucune garantie d'exhaustivité en raison de sa nature épuisante pour le traducteur. L'automatisation de cette démarche rendrait un fier service aux praticiens et pourrait prendre la forme d'un repérage informatisé fournissant à la fois les termes en ordre alphabétique, en ordre d'apparition dans le texte et sous forme de listes KWIC, c'est-à-dire dotés de contextes.

À cet égard, il est bon de rappeler les arguments présentés par le groupe de recherche de l'Université Carnegie-Mellon :

Toutefois, des améliorations sont encore possibles afin de simplifier d'autres aspects du processus de traduction, en permettant notamment :

1. Le relevé simultané des contextes de toutes les occurrences d'un terme donné dans le document de départ. Ceci élimine les consultations répétitives après chaque apparition du terme dans le texte, ainsi que le retour en arrière pour modifier des équivalents lorsque, par la suite, on en découvre de meilleurs.

2. L'impression, après révision, du texte d'arrivée, ce qui supprime la frappe finale de la traduction achevée. Cette frappe finale constitue un problème particulièrement aigu, car il est rare que les traducteurs aient à leur disposition des secrétaires bilingues, ce qui les entraîne à une importante perte de temps au contrôle et à la correction du texte frappé pour eux.

Ces processus peuvent être incorporés à une banque de terminologie en les intégrant à un système de traduction qui permette une interaction avec un dictionnaire et un programme d'édition. Le traducteur travaillerait directement avec l'ordinateur, éditant les textes dans les langues de départ et d'arrivée, assisté par cet énorme dictionnaire multilingue plus rapidement accessible que la grande collection de documents que possèdent la plupart des traducteurs ${ }^{13}$.

Pour le traducteur qui préfère travailler au terminal, on conçoit aisément deux modes de présentation du texte de départ. D'abord, la mise au point d'un programme utilitaire conçu pour la présentation du texte en mode dialogué permettrait au traducteur de travailler sur sa version sans recourir à d'autres supports comme le papier ou le dictaphone. Pour être véritablement utile, ce programme devrait incorporer plusieurs fonctions inédites telles la présentation

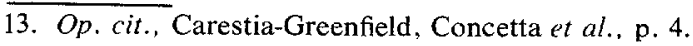


de contextes suffisamment complets, la consultation des sources documentaires sans avoir à effacer le contenu de l'écran et la communication en direct avec des collègues traducteurs.

Une deuxième innovation, encore plus révolutionnaire, se situe au niveau des systèmes de synthèse et de reconnaissance de la voix humaine ${ }^{14}$. Dans quelques années, lorsqu'ils seront opérationnels, l'application de ces systèmes à la traduction donnera aux traducteurs la possibilité de travailler sur leurs textes de la même manière qu'ils le font déjà à l'aide du dictaphone. Quand on connaît la popularité de cet instrument chez les traducteurs professionnels, le jumelage futur de la télématique et du dictaphone nous laisse entrevoir des répercussions importantes pour la traduction commerciale.

Dans cette marche vers l'informatisation des fonctions du processus de la traduction, l'étape ultime et sans aucun doute la plus difficile à réaliser est celle de la traduction automatique. Bien que ce sujet déborde la présente étude, les recherches en traduction automatique ont produit nombre de réalisations techniques qui peuvent nous intéresser.

Une des réalisations qui retient particulièrement notre attention est la mise au point de systèmes de reconnaissance de structures syntaxiques. Conçus à l'origine pour découvrir la structure profonde des phrases en vue de les traduire en langue d'arrivée, ces instruments ne semblent pas avoir été appliqués en dehors du cadre précis pour lequel on les a développés. Présentement, nous n'avons pas l'impression qu'on envisage la possibilité d'utiliser un tel outil comme réviseur automatique de textes plutôt que comme traducteur, ce qui pourrait être une aide précieuse au processus de la traduction. Outre la fonction de "contrôleur syntaxique», on conçoit aisément l'addition d'autres fonctions comme le contrôle de l'orthographe, des lettres majuscules et de la ponctuation ou encore l'élaboration de programmes de tri et d'indexation pour la mise en forme de documents volumineux. De plus, le jumelage du contrôleur syntaxique avec un dictionnaire automatisé permettrait une vérification de la terminologie.

Quand on connaît les exigences de la révision des textes, on apprécie encore plus l'idée de pouvoir se fier à un appareil qui s'occuperait des aspects mécaniques de cette besogne. L'attention ou la concentration du réviseur ne peut jamais rester constante d'un bout à l'autre de son texte. La recherche d'une tournure défectueuse, d'une ponctuation maladroite, de l'orthographe erronée, voilà autant de facteurs qui rendent le travail du réviseur analogue à celui de fouiller dans une botte de foin pour trouver cette célèbre aiguille! La révision assistée par ordinateur pourrait être un autre bienfait de la recherche en traductologie.

Enfin, si l'heure de la traduction automatique n'est pas encore venue, elle approche. L'époque est révolue où l'on pouvait affirmer impunément que la traduction automatique était un rêve utopique, ou du moins futuriste... qu'elle

14. Haton, Jean-Paul, "Reconnaissance de la parole : état des recherches», in Zéro un informatique, mensuel, $\mathrm{n}^{\circ} 131$, juin/juillet 1979 , p. 44-61. 
demeurerait pour longtemps encore la chasse gardée des chercheurs universitaires.

Ce tour d'horizon des contributions de l'informatique à la traduction constitue une première tentative pour établir une vue d'ensemble, une synthèse des besoins d'une pratique de la traduction qui reposerait sur un support informatique. Pour résumer ces nombreux propos, nous nous joignons au groupe de l'Université Carnegie-Mellon pour souligner la pertinence des recommandations du rapport ALPAC.

Il semble que la recherche du dialogue traducteur-ordinateur ait été négligée et qu'aucune étude concernant le processus de traduction n'ait été faite. En outre, les systèmes interactifs de traduction n'ont pas été éprouvés et, par conséquent, il n'existe aucune donnée exprimée en termes d'efficacité des coûts et d'amélioration de la qualité.

Il peut être utile de rappeler ici les recommandations du rapport ALPAC qui constitue encore un guide pour la recherche dans ces domaines négligés. Ce rapport conseille, entre autres, d'orienter la recherche vers:

1. Le processus cognitif de la traduction;

2. Les manières d'accélérer ce processus;

3. Les retards de l'ensemble du processus de traduction et les moyens d'éliminer ces retards aussi bien dans les publications qu'au niveau individuel;

4. La vitesse et le coût relatifs de différentes méthodes de traduction assistée par ordinateur;

5. Les différentes façons d'adapter au processus de la traduction, les possibilités d'impression et de mise en forme automatique;

6. La production de travaux de référence adéquats pour le traducteur, y compris l'adoption de glossaires existants, principalement pour la consultation automatique d'un dictionnaire dans une traduction assistée par ordinateur ${ }^{15}$.

En guise de conclusion, quand bien même on mettrait l'équipement le plus perfectionné à la disposition du traducteur, si ce dernier n'est pas conscient des exigences culturelles de sa profession, il pourra difficilement prétendre être un véritable traducteur. Les aides informatisées ne feront qu'accroître la pertinence de ce constat car l'automatisation ne pouvant s'appliquer au processus cognitif de la traduction, elle finira par mettre ce dernier en relief plus que jamais auparavant. On peut aisément affirmer que les deux grands bastions du traducteur se situent dans sa connaissance profonde des langues et des cultures. Plus on mécanise l'activité traduisante, plus on diminue l'importance de l'intervention humaine grâce à l'entrée en mémoire des données répétitives. En conséquence, on peut déjà prévoir que la nouvelle génération des traducteurs sera faite de personnes plus éveillées, plus compétentes et peut-être même plus compétitives entre elles.

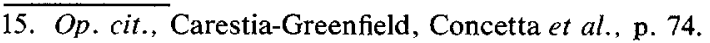




\section{BIBLIOGRAPHIE}

ANDREYEWSKY, Alexander et Donald McCRACKEN, Automating Translation in the 1980's. Pittsburgh (Pennsylvanie), Carnegie-Mellon University, août 1979, $10 \mathrm{p}$.

ALPAC, Languages and Machines: Computers in Translation and Linguistics, Report by the Automatic Language Processing Advisory Committee, Division of Behavioral Sciences, National Academy of Sciences, National Research Council, Publication 1416, Washington (D.C.), 1966.

ARTHERN, P. J., «Machine Translation and Computerized Terminology Systems : a Translator's Viewpoint», in Snell, 1979, p. 77-108.

BERGERON, Viateur et al., Lexicographie, bilinguisme juridique et ordinateur, Ottawa, Les éditions de l'Université d'Ottawa, 1976, $330 \mathrm{p}$.

BERGERON, Viateur et al., Un lexique bilingue de 1001 mots extraits de 24 chapitres des statuts révisés du Canada, Ottawa, Université d'Ottawa, 1975, 324 p.

Bl_UMENTHAL, R., "Testing out two Pocket Electronic Translators", in New York Times, March 25, 1979.

BRUDERER, H. E., Handbook of Machine Translation and Machine-Aided Translation: Automatic Translation of Natural Languages and Multi-Lingual Terminology Data Banks, Amsterdam, North-Holland, 1977.

CANISIUS, P., «Automatic Partial Transiation in a Multilingual Information System», in CEC, vol. 1, 1977, p. 259-269.

CARESTIA-GREENFIELD, Concetta et Daniel SERAIN, la Traduction assistée par ordinateur; des banques de terminologie aux systèmes interactifs de traduction, Paris, AFTERM, août $1977,116 \mathrm{p}$.

CEC (Commission of the European Communities), Overcoming the Language Barrier (Third European Congress on Information Systems and Networks), Luxembourg, mai 1977.

CHOUINARD, Rachel, Vers une nouvelle génération de traducteurs-médiatiseurs, Québec, Université du Québec, $12 \mathrm{p}$.

DUBUC, R., Description du système TERMIUM, Montréal, Université de Montréal - Banque de terminologie, 1979.

DUCROT, J. M., Research for an Automatic Translation System for the Diffusion of Scientific and Technical Textile Documentation in English Speaking Countries, Final Report, BoulogneBillancourt, Institut Textile de France, 1972.

DUMAS, Pierre et al., l'Adaptation française de PLATON, Québec, Université du Québec, 1977, $53 \mathrm{p}$.

ELLISTON, J.S.G., "Computer Aided Translation: a Business Viewpoint", in Snell, 1979, p. $149-158$.

FORTIN, J. M., la Banque de terminologie du Québec, Québec, Office de la langue française, 1974.

GAGNÉ, Francine, "Terminoq II", in META, Journal des traducteurs, numéro spécial : La documentation, vol. $25, \mathrm{n}^{\circ} 1$, mars 1980, p. 182-186.

GOETSCHALCKX, J., "Terminological Activities in the European Institutions, with special reference to EURODICAUTOM", in CEC, vol. 1, 1977, p. 123-152.

HATON, Jean-Paul, "Reconnaissance de la parole : état des recherches", in Zéro un informatique, mensuel, $\mathrm{n}^{\circ} 131$, juin/juillet 1979, p. 44-51.

HUTCHINS, W. J., "Machine Translation and Machine Aided Translation", in Journal of Documentation, $\mathrm{n}^{\circ}$ 34, 1978, p. 119-159.

JAUBERT, Y.-P. et François DUMAS, Rapport sur l'utilisation de machines de traitement de mots comme aides à la traduction (rapport interne non publié), Ottawa, Secrétariat d'État, novembre $1979,50 \mathrm{p}$.

KAHN. Annie, "Weidner ou la TAO (Traduction assistée par ordinateur)", in Zéro un informatique, hebdomadaire, $\mathrm{n}^{\circ} 582,3$ mars 1980 , p. 6.

KROLLMANN, F., "Linguistic Data Banks and the Technical Translator", in META, Journal des traducteurs, vol. 16, 1971, p. 117-124.

KROLLMANN, F., "User Aspects of An Automatic Aid to Translation as Employed in a Large Translation Service", in CEC, vol. 1, 1977, p. 243-257. 
KROLLMANN, F., H. SCHUCK et U. WINKLER, Herstellung textbezogener Fachwortlisten mit einem Digitalrechner : ein Verfahren der automatischen Ubersetzungshilfe, Beiträge zur Sprachkunde und Informationsverarbeitung, $\mathrm{n}^{\circ} 5,1965$, p. 7-30

LAWSON, V., "Tigers and Polar Bears, or: Translating and the Computer", in The Incorporated Linguist, $\mathrm{n}^{\circ} 18,1979$, p. 81-85.

LIPPMANN, E. O., On-line Generation of Terminological Digests in Language Translation: an Aid in Terminology Processing, IBM Research Communication, septembre 1975.

MALIK, Rex, "Le système d'information juridique Eurolex sera accessible par Euronet depuis le continent", in Zéro un informatique, hebdomadaire, $n^{\circ}$ 586, 31 mars 1980, p. 26.

PARE, M., la Banque de terminologie de l'Université de Montréal, Nice, Fédération internationale des traducteurs, 1974.

REVUE JURIDIQUE THÉMIS, "Jurimétrie : informatique juridique", réd. en chef : R. Le Hir, 1971, 6 R.J.T., $\mathbf{n}^{\circ} 1$.

REVUE JURIDIQUE THÉMIS, "Informatique juridique", directeur général : Francis Rigaldies, 1976, 11 R.J.T., nos 1 et 2.

RONDEAU, G., J.-F. GRÉGOIRE et P. TESSIER, "Banques de terminologie et linguistique computationnelle", in META, Journal des traducteurs, vol. 22, 1977, p. 184-191.

SAGER, J. C., "Multilingual Communication: Chairman's Introductory Review of Translating and the Computer", in Snell, 1979, p. 1-25.

SAGER, J. C., "The Computer and Multilingualism at the European Commission", in Lebende Sprachen, $\mathrm{n}^{\circ}$ 3, 1979, p. 103-107.

SAGER, J. C. et R. L. JOHNSON, "Terminology: the State of the Art", in AlLA Bulletin, $\mathrm{n}^{\circ} 22$, 1978, p. 1-12.

SCHULZ, J., "A Terminology Data Bank for Translators (TEAM)", in META, Journal des traducteurs, vol. $25, \mathrm{n}^{\circ} 2$, juin 1980, p. 211-229.

SCHWAB, Wallace J., "La réalisation du thésaurus-s et du thésaurus-g", in la Revue juridique Thémis, n 1, 6 R.J.T., 1971, p. 69-84.

SCHWAB, Wallace, la Traduction et le système PLATON, Guide $n^{\circ} 17$, Université du Québec, Vice-présidence aux communications, 1979.

SCHWAB, Wallace et Richard ST-DENIS, "PLATERM" : la banque de terminologie du système PLATON", in META, Journal des traducteurs, vol. 25, $\mathrm{n}^{\circ}$ 3, septembre 1980.

SOMERS, H. L. et J. McNAUGHT, "The Translator as a Computer User", in The Incorporated Linguist, Londres, vol. 19, $\mathrm{n}^{\circ} 2$, printemps 1980.

TANKE, E. H., "Implementing Machine Aids to Translation" ; in Snell, 1979, p. 45-69.

TAUM, "Le système de traduction automatique de l'Université de Montréal (TAUM)", in META, Journal des traducteurs, vol.18, n 3, 1973, p. 227-289.

TAUM, Groupe de recherche en Traduction Automatique de l'Université de Montréal (TAUM), Montréal, TAUM, 1979.

TOMA, P., "An Operational Machine Translation System", in Brislin, New York, Gardner Press, 1976, p. 247-259.

WOHL, Amy, "A Review Office Automation", in DATAMATION, février 1980, vol. 26, $\mathrm{n}^{\circ} 2$, p. 116-119.

Zéro un informatique, hebdomadaire, IBM : DTAF progiciel de traduction assistée par ordinateur, $\mathrm{n}^{\circ} 588,14$ avril 1980 . 